\title{
Experimental Study on the Performance of Vortex Microbubble Generator
}

\author{
Zhekun $\mathrm{Li}^{1, \mathrm{a}^{*}}$, Wei He $\mathrm{H}^{1, \mathrm{~b}}$, Wenli Shi ${ }^{1, \mathrm{c}}$ and Yujin $\mathrm{Fan}^{1, \mathrm{~d}}$ \\ ${ }^{1}$ School of Mechanical and Electrical Engineering, Kunming University of Science and Technology, \\ Kunming 650500, China \\ azhekunlikust@sina.com, bhw_big@126.com, 'swl_ie@163.com, dfanyujinkmust@163.com
}

Keywords: microbubble generator; performance test; working condition; microbubble

Abstract. Based on the analysis of structure and working principle of the vortex microbubble generator, the generator's prototype is made and the experiment system is established by us. Under different working conditions the experimental researches on the performance have been done. A better working condition has been found. The paper provides good reference for the experimental study on the performance of the vortex microbubble generator.

\section{Introduction}

Even though there is a reasonable structure design of a microbubble generator, the theoretical analysis of the system can not replace the physical experimental verification. Through the experiments, we can prove the correctness of theoretical analysis, computer simulation and machining, find the actual operating conditions, and provide important technical reference for the practical application of this kind of microbubble generator.

\section{The Structure and Working Principle of the Vortex Microbubble Generator}

The structure of the vortex microbubble generator has a decisive effect on the performance of the microbubbles generation [1], while the size, number and distribution of the microbubbles have a great influence on the effect of water treatment.

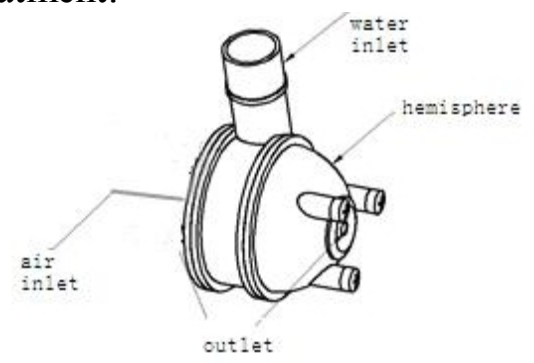

Fig. 1 Schematic Diagram of the Vortex Microbubble Generator Made by Us

Fig. 1 is the schematic diagram of the vortex microbubble generator [2]. The fluid with a certain initial velocity along the tangential direction from the water inlet flows into the microbubble generator, and then produces vortex flow inside. According to the conservation of momentum moment and Bernoulli equation:

$$
u_{\partial} r=C
$$

$u_{\partial}$ is the fluid flow velocity in the tangential direction, $\mathrm{m} / \mathrm{s} ; \mathrm{r}$ is the radius of rotation of the fluid particle, $\mathrm{m}$;

$\mathrm{C}$ is the constant of the moment momentum.

$$
p+\frac{1}{2} \rho v^{2}+\rho g h=c
$$

$\mathrm{p}$ is fluid pressure, MPa; $\rho$ is the density of fluid, $\mathrm{kg} / \mathrm{m}^{3} ; \mathrm{v}$ is velocity, $\mathrm{m} / \mathrm{s} ; \mathrm{h}$ is vertical height, $\mathrm{m}$; $\mathrm{g}$ is acceleration of gravity, $\mathrm{m} / \mathrm{s}^{2} ; \mathrm{C}$ is a constant, dimensionless. 
In ideal conditions, if the energy loss of the fluid is not considered and the flow of the fluid is always in the laminar flow, the fluid flows from the tangential direction into the inside of the microbubble generator, the moment momentum keeps in a constant, as the point is more close to the center, its radius of rotation is less and the tangential velocity is larger. With the continuous variation of tangential velocity, it can be known from the Bernoulli equation, the pressure energy will convert into kinetic energy, the point is more close to the center, the tangential velocity is larger and larger, the pressure will become smaller and smaller. A negative pressure zone will be generated near the middle axis zone of the microbubble generator. At the negative pressure, the air will be sucked into the vortex microbubble generator from the air suction nozzle (air inlet) and then go out from the mixture nozzle outlet due to different diameters of the inlet and the outlet, and the negative pressure values at both sides are also different, so that will produce in the middle axis of a pressure gradient. The sucked air will be mixed and broken into microbubbles and then sprayed out from the mixture outlet.

\section{The Physical Manufacturing Process of the Vortex Microbubble Generator}

The generator's inner chamber diameter and the ring diameter respectively are $50 \mathrm{~mm}$, water inlet diameter is $12 \mathrm{~mm}$, air inlet diameter is $3 \mathrm{~mm}$ and mixture outlet diameter is $6 \mathrm{~mm}$. The 304 stainless steel pipe and hemisphere are selected. The specific parameters are shown in Table 1.

Table1 Specific Parameters of the Generator Parts

\begin{tabular}{|c|c|c|c|}
\hline Parts & Inner Diameter $(\mathrm{mm})$ & Wall Thickness $(\mathrm{mm})$ & Length $(\mathrm{mm})$ \\
\hline Ring & 50 & 1.5 & 20 \\
\hline Hemisphere & 50 & 1.5 & - \\
\hline Water Inlet pipe & 12 & 1 & 35 \\
\hline
\end{tabular}

Manufacturing process description:

Because the water inlet pipe and the ring are tangential in the intersection, a numerical control (NC) machine is used to manufacture the intersection on the ring part first. Then the water inlet pipe is inserted into the ring and fixed by the argon arc welding, the excess portion of the water inlet pipe is cut along the inner wall of the ring chamber by an electrical wire cutting machine. Finally, the hemisphere drilled a mixture outlet and a round stainless steel plate drilled an air inlet fixed with the ring at two ends by the argon arc welding. A physical prototype of the vortex microbubble generator is got as shown in Fig. 2.

The vortex microbubble generator is connected by a hose with water pump, in the middle it is connected with a relief valve, a three-way valve, and a liquid flow meter etc..

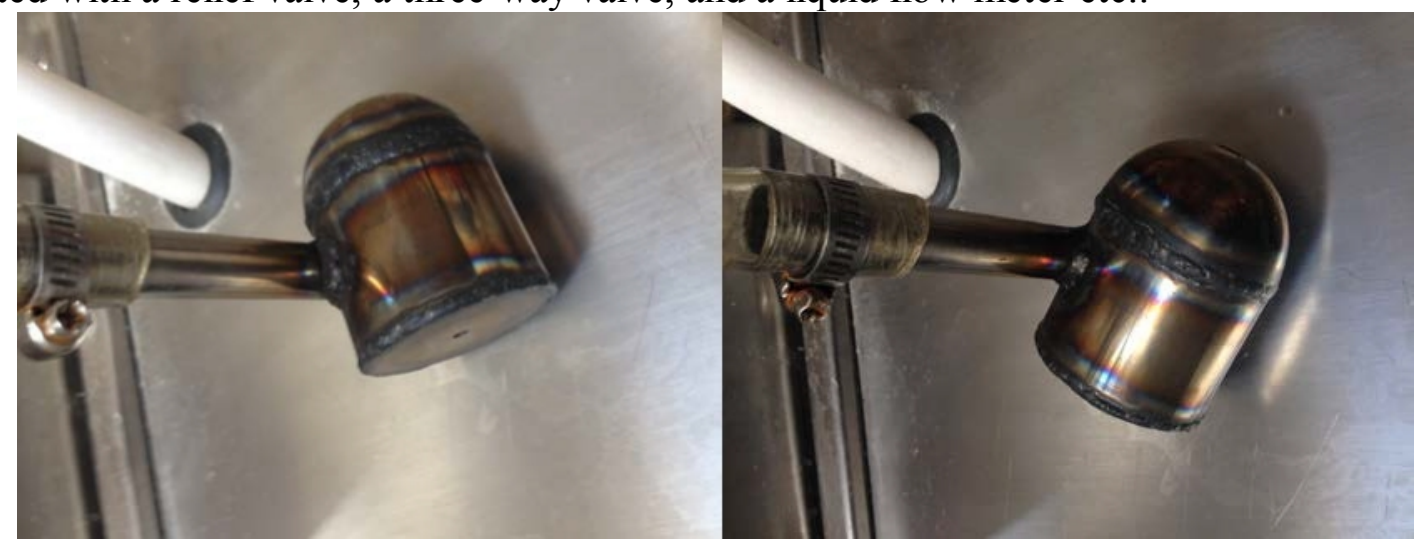

Fig. 2 Physical Prototype of the Vortex Microbubble Generator

\section{Experimental Principle and Device}

The experimental apparatus used in the experiments include: the vortex microbubble generator, pump, pressure gauge, flow meter, three-way valve, pressure relief valve, container, 500 times USB microscope. 
The high-pressure pump pressurizes water into the vortex microbubble generator and the inside vortex flow is generated, the negative pressure at the air inlet is generated too. The air is sucked from a small diameter hose spreading out of the water surface, and is sheared and broken into tiny air bubbles in the vortex microbubble generator, and then the microbubbles are mixed with the water and ejected from the mixture outlet into the flotation column. In the experiment the flotation column is made by the transparent organic glass that can help us to observe the process of generating microbubbles. At the entrance of the microbubble generator a flow meter is installed. The inlet pressure is set by a pressure relief valve and the flow is regulated by a three-way valve, after that we can get different inlet velocity to obtain different experimental results. When the water flow in the flotation column increases to the observation channel and the valve will be closed, the bubbles in the groove are stable, and can be photographed on the computer by 500 times.

\section{The Relationship between the Sizes of the Microbubble and Working Condition Parameters}

In the experiment the pressure, the flux and the velocity can be regulated for the experimental requirements. At present, there are two methods that are widely used to measure the size and distribution of the bubbles. One is to measure the size and distribution of bubbles by means of electronic probe technique, which is used not so widely. Other is the acoustic or optical detection technology to measure the size of the bubble and distribution [3], which is used more widely. In this paper a USB microscope with high magnification takes pictures of bubbles and transmits them into AutoCAD software to measure bubble diameter and distribution number. In order to reduce the error of photographic exposure, the bubbles will stay in the observation slots until all the bubbles are in the stable stationary state, the photographs are taken and at the same inlet velocity we take multiple shootings. An average value is calculated out in order to reduce the experimental error.

Fig. 3 is a ruler with a 500 times USB microscope and the smallest unit is $0.1 \mathrm{~mm}$. In order to get a high statistical accuracy, the photos are transmitted into the AUTOCAD software, in the size of the $1 * 1 \mathrm{~mm}$ area there are average 20 bubbles, in the same inlet flow, the observation slot is emptied and filled 3 times, the 3 measurements are repeated, so one diameter size of bubbles has measured about 60 bubble diameters.

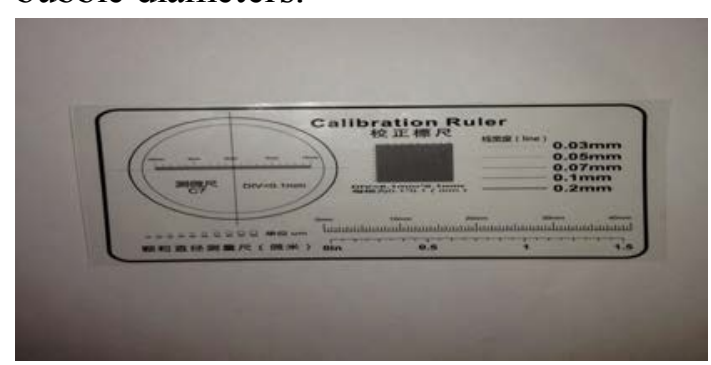

a) Measuring Ruler

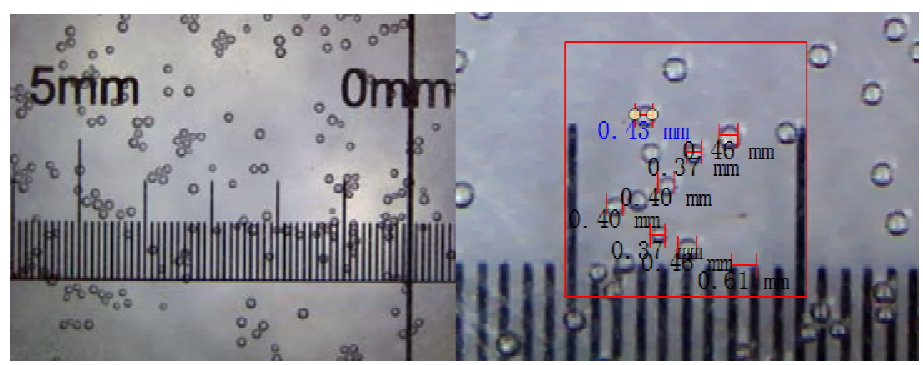

b) Measuring Principle

Fig. 3 Rule and Diagram of Microbubble Diameter Measuring

The main parameters in the experiment are the inlet water flow and air flow, and the water flow velocity can be calculated. Inlet flow flux adjustment range is 30 50 times per minute and the air flow flux adjustment range is 4 22 times per minute. The average diameters of the bubbles measured by different adjusting operating conditions are shown in Table 3 . When the air inlet is fully open, the water flow fluxes and the air flow fluxes are respectively: 30:14, 40:17 and 50:22.

Table.2 Average Diameters of Microbubbles under Different Working Conditions (mm)

\begin{tabular}{|c|c|c|c|c|}
\hline \multicolumn{2}{|c|}{} & \multicolumn{3}{|c|}{ Air Flow Flux } \\
\cline { 3 - 5 } \multicolumn{2}{|c|}{} & fully open & 13 & 4 \\
\hline \multirow{3}{*}{$\begin{array}{c}\text { Water Flow } \\
\text { Flux }\end{array}$} & 30 & 643 & 617 & 552 \\
\cline { 2 - 5 } & 40 & 526 & 575 & 534 \\
\cline { 2 - 5 } & 50 & 471 & 434 & 398 \\
\hline
\end{tabular}




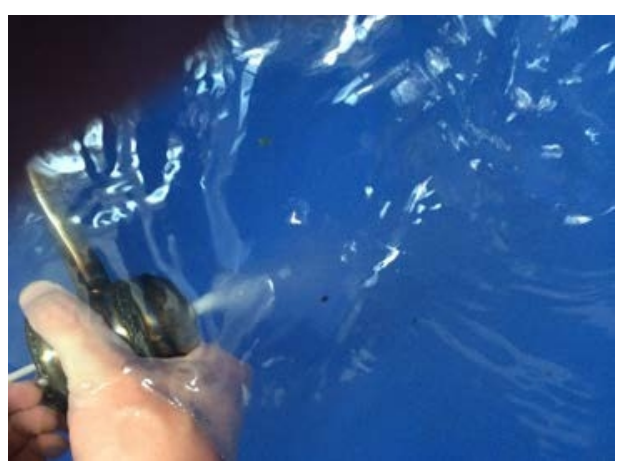

Fig. 4 Experiment Photo

The percentage distributions of microbubbles under different water flow flux and different air flow flux are observed. A picture of the bubbling process in the experiment is shown in Fig. 4.

By the experiments, we found that the average diameter of the microbubbles is smaller when the air flow flux is constant and the water flow flux gradually increases. In principle, when the inlet fluid flow is larger, it indicates a larger inlet velocity, corresponding in the tangential velocity is larger and the shear force is stronger, it is benefit for air smashing and the size of microbubble diameter is much smaller. Otherwise as the inlet water flow flux is constant, the diameter of the microbubble can reduce with the small air flow flux. The reason for this phenomenon is that the smaller of the air flow flux per unit time, the air will have long time and high probability to be mixed and broken into microbubbles and the average bubble diameter is smaller. Through observation of several groups of microbubble diameter distributions from the experiments, as the water flow flux is 40 , the air flow flux is 13 , the microbubble diameter between the $40-80 \mu \mathrm{m}$ content is higher and reaches $75 \%$, and the average diameter of microbubble is $57.5 \mu \mathrm{m}$, the content of diameters larger than $80 \mu \mathrm{m}$ is lower, it reaches the requirement of diameter and number of microbubble for water treatment.

\section{Conclusion}

This paper established the performance experiment system for the vortex microbubble generator. On the self-made microbubble generator prototype, under different working conditions the experimental studies have done. The results confirm that the theoretical analysis of the microbubble generator is correct, the computer simulation study is effective, and the structure design is reasonable. Under certain conditions, the vortex microbubble generator can obtain better sucking effect, the paper provides the important reference for the practical application of the microbubble generator.

\section{Acknowledgements}

This work was sponsored by China Natural Science Foundation Project (No. 51168020) and Yunnan Province Natural Science Foundation Project (No. 2013FZ024). We will thank here!

\section{References}

[1] Li Zhekun, Li Min, Qiang Yuanli. Study on microbubble generators for treatment of wastewaters. 5th International Conference on Responsive Manufacturing - Green - Manufacturing, ICRM 2010, Vol 2010, No 565, pp 488-493.

[2] Deng Jiushuai, Li Zhekun, He Wei, Yu Guorui, Fan Yujin, Shi Wenli, He Banggui. A vortex microbubble generator. Patent number: 2014203772964, 2015.01.28

[3] Collins G L, Jameson G J. Double layer Effects in the Flotation of the Particles. Chem. Eng. Sci., 1977, (32), pp239-246. 